\title{
NISTIR 88-3867
}

\section{Report on Cabling Navy Bases for Voice, Data and Video Appendix A: Premises Wiring Plan for Data, Voice and Video}

\author{
W. E. Burr and A. L. Koenig \\ U.S. DEPARTMENT OF COMMERCE \\ National Institute of Standards and Technology \\ (Formerly National Bureau of Standards) \\ National Computer and Telecommunications Laboratory \\ Advanced Systems Division \\ Gaithersburg, MD 20899
}

August 1988

Issued December 1988

FE GPY

Sponsored by:

Naval Data Automation Command

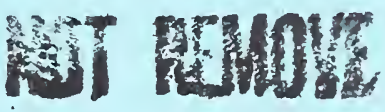

Washington Navy Yard

Washington, DC 20374

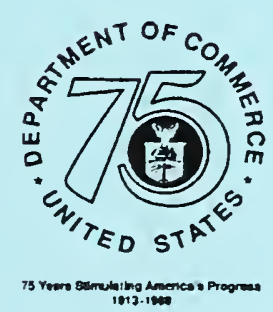





\section{Report on Cabling Navy Bases for Voice, Data and Video Appendix A: Premises Wiring Plan for Data, Voice and Video}

W. E. Burr and A. L. Koenig

U.S. DEPARTMENT OF COMMERCE

National Institute of Standards and Technology

(Formerly National Bureau of Standards)

National Computer and Telecommunications Laboratory

Advanced Systems Division

Gaithersburg, MD 20899

August 1988

Issued December 1988

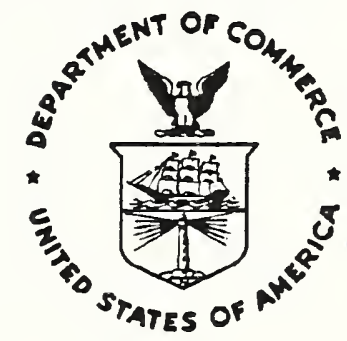

Sponsored by:

Naval Data Automation Command

Washington Navy Yard

Washington, DC 20374
National Bureau of Standards became the

National Institute of Standards and Technology

on August 23, 1988, when the Omnibus Trade and

Competitiveness Act was signed. NIST retains

all NBS functions. Its new programs will encourage

improved use of technology by U.S. industry.

\section{U.S. DEPARTMENT OF COMMERCE \\ C. William Verity, Secretary}

NATIONAL INSTITUTE OF STANDARDS

AND TECHNOLOGY

Ernest Ambler, Director 

TABLE OF CONTENTS

Page

1. Introduction 1

2. Applications 2

2.1 Voice 2

2.1.1 Analog Voice 2

2.1 .2 Digital (ISDN) 2

2.2 Data 3

2.2 .1 Terminals 3

2.2 .2 Workstations and PCs 4

2.2.3 Mini's and Mainframe Computers 5

2.3 Storage Servers 5

2.3.1 Image Processing 6

2.4 Video

3. Networks

3.1 Local Area Networks (LANs) 7

3.1 .1 Topologies

3.1 .2 Access Methods 8

3.1 .3 Standards 8

3.2 Wide Area Networks 10

4. Media 10

4.1 Electrical Media 10

4.1.1 Twisted Pair 10

4.1.2 Coaxial Cable 11

4.2 Optical Media 12

4.2.1 Multimode Fiber 13

4.2.2 Single Mode Fiber 14

5. Commercial Wiring Plans 15

5.1 IBM Cabling System 15

5.2 AT\&T Premises Distribution System (PDS) 16

5.3 DECconnect 18

6. Recent Developments in Wiring Technology 19

6.1 Unshielded Twisted Pair for IEEE 802.3
Applications

6.2 Standards Committee work 21

6.3 New Wire Types 22

7. Conclusion 22

8. References 23

$\begin{array}{lll}\text { APPENDIX A. Premises Wiring Plan for } & \\ & \text { Data, Voice and Video }\end{array}$

CONTRIBUTION OF THE NATIONAL BUREAU OF STANDARDS. NOT SUBJECT TO COPYRIGHT . 



\section{REPORT ON CABLING NAVY BASES FOR VOICE, DATA AND VIDEO}

\section{Introduction}

The fundamental premise of this study and plan is that administrative communications needs of the Navy as a whole are not dramatically different than those of other large organizations in the Federal Government and industry, and that they will be satisfied by the same methods and technologies that prevail commercially in the United states. The focus of the effort therefore has not been a study of the particular requirements of the Navy, rather it has been to addresses the communications technologies expected to dominate in the 1990s and the commercial wiring plans which have been devised to accommodate them.

This plan proposes a common approach to wiring Navy bases for voice, data and television for the remainder of this century. It is based upon the concept of a base cable plant which enters each building into a master wire center. This wire center is then connected by trunks to secondary wire closets strategically distributed throughout the building. Individual users are then connected to the secondary wire closets. All buildings are connected by trunks to the base master wire center.

For voice and data, two media will be pervasive: copper twisted pairs and multimode optical fiber waveguides. It is anticipated that all voice and data connection between buildings through the external base cable plant will be via fiber. Both fiber and copper twisted pair trunks will be used to connect the building master wire center to secondary wire closets. A four twisted pair Integrated Services Digital Network (ISDN) compatible copper cable will be run from the secondary wire closets to every individual workstation.

The twisted pair cable will accommodate ISDN compatible digital voice and data services, some Local Area Network (LAN) equipment, many existing asynchronous terminal's operation at rates up to 19.2 kbaud, and conventional analog voice equipment. Many LANs, however, may require either co-axial cable, special shielded twisted pairs or optical fiber connections to secondary wire centers. No reasonable wiring plan could accommodate every needed variation, so this LAN specific work station to wiring closet cabling will be installed on an as needed basis.

Intra-building trunking of either voice or data between wiring closets will be via either twisted pair or optical fiber. Virtually all LANs can now be accommodated on optical fibers, and the only reason for not carrying fiber all the way to every 
workstation is economic; the station interfaces to shielded twisted pair or co-axial cable are significantly less expensive. All inter-building trunking of either voice or data will be via multimode optical fiber.

In many respects this is similar to what has been done for many years in the wiring of buildings and campuses. The major change from past practice is the pervasive use of optical fiber as the main trunking medium, and, largely in consequence of this, a significant increase in the amount of active electronics, multiplexors and the like, now required in the wiring closets to serve the fiber.

Video services, however, will use coaxial cable. This is because analog National Television System Committee (NTSC) compatible fiber optic equipment is not at present cost competitive with well proven conventional cable TV technology, and it is not anticipated that high resolution digital video, which has been widely discussed as a possible future fiber application, will become a widespread commercial reality in the United states in this century. In other words, nothing is expected to supplant the huge installed base of analog NTSC TVs before the year 2000. Two coaxial cable buses, one in and one out, will connect wire centers to the basewide cable TV distribution system.

2. Applications

2.1 Voice

\subsubsection{Analog Voice}

The Navy has a large installed base of conventional analog telephones, using twisted pair copper media. Much of this equipment will remain in service for many years. It is expected, however, that new private branch exchange (PBX) installations will primarily be for digital ISDN compatible equipment.

\section{1 .2 Digital (ISDN)}

Telephone carriers are planning to offer (and in some cases are now offering at least preliminary versions of) a new worldwide standard digital telephone service called the Integrated Services Digital Network (ISDN). Digital transmission and switching is now widely used in central offices and in the carrier trunk networks. At present, since most instruments are analog, the conversion to digital takes place at network switching equipment. The advantage to digital switching of voice is that the addition of noise in each successive link can be eliminated and the signal can transit many successive links in the network without any degradation 
in quality. In the future, the analog to digital conversion will take place in the telephone instrument itself. This will permit comparatively high rate transmission of digital data as well as voice direct to any telephone instrument.

ISDN defines two digital services. The basic rate service includes two $64 \mathrm{kbit} / \mathrm{s}$ voice/data channels (called "B" channels) plus a $16 \mathrm{kbit} / \mathrm{s}$ signalling channel (called a "D" channel). This basic service is sometimes referred to as a $2 B+D$ service and allows full duplex operation over a single twisted pair line. Each $B$ channel allows either a fully digital voice call or $64 \mathrm{kbit} / \mathrm{s}$ data service; the D channel carries signalling for call set-up or, optionally, packetized data. The "Primary" rate service varies between North America and Europe. In North America the Primary rate is $1.536 \mathrm{Mbit} / \mathrm{s}$ for compatibility with the installed Tl carrier system at this rate. This is considered to be twenty three B channels (at $64 \mathrm{kbit} / \mathrm{s}$ ) plus one D channel (at $64 \mathrm{kbit} / \mathrm{s}$ ). In Europe the primary rate is $2.048 \mathrm{Mbit} / \mathrm{s}$ or $30 \mathrm{~B}+\mathrm{D}$.

Since ISDN facilities will eventually be pervasive, and since the ISDN Basic full duplex service at $64 \mathrm{kbit} / \mathrm{s}$ is a dramatic improvement over the use of 2400 or 4800 baud modems over the present telephone network, it is expected that ISDN will become, during the 1990's, the primary vehicle by which the Navy and most of the rest of the country conduct long distance digital communications between remote locations. In addition, ISDN will provide higher rates even for local terminal applications. Integrated Voice/Data (IVD) terminals using the $64 \mathrm{kbit} / \mathrm{s}$ rate will eventually be commonplace.

At some point in the 1990's ISDN services for switching the Primary rate of $1.536 \mathrm{Mbit} / \mathrm{s}$ may become widespread in the public networks and in private branch exchanges. If so, this will somewhat extend the utility of ISDN for computer to computer or computer to storage server applications.

2.2 Data

\section{2 .1 Terminals}

The Navy and other users have a large inventory of terminals. Many of these are "standard" ASCII terminals, which are derived originally from teletypes and use asynchronous transmission of the ASCII line codes at typical rates of $300,1200,2400,4800,9600$ 
and 19200 baud (this roughly corresponds to 30, 120, 240,480 , 960 and 1920 ASCII characters per second). These terminals generally have an RS-232 interface and are usually hooked to computers by twisted pair wires, or to modems for connection through the public telephone network.

A number of other types of terminals exist, and many of these, particularly the bit synchronous IBM 3360 type terminals, operate at higher rates and use either coaxial cable or shielded twisted pair media. Another fairly common type of terminal is the vector graphics terminal. It is expected that these mainframe to terminal graphics applications will eventually be largely replaced by workstations with the local processing power for complex image manipulation.

Conventional asynchronous terminals will initially be attached to ISDN or to LANs through adapters, which will let them operate at their highest rate (generally 9600 or 19200 baud), but may, in time, be largely supplanted by terminals designed to utilize the higher ISDN $64 \mathrm{kbit} / \mathrm{s}$ rate and to attach directly to the ISDN network, or by personal computers and workstations, which can be attached directly to the ISDN network or to a LAN.

\subsubsection{Workstations and PCs}

Workstations and personal computers are physically small, but often quite powerful, computers, which are generally operated by a single user. They are supplanting "dumb" terminals connected to time sharing computers in many applications, and are largely taking over graphics applications. There is no clear distinction between a PC and a workstation, however; the term workstation usually implying a more powerful computer and a high resolution graphic display. Many PCs and workstations have internal hard disks, but there is a trend to "diskless" workstations. Whether or not a PC or workstation has an internal hard disk, there is a need in many applications to attach many PCs or workstations to a large, shared data base. In these applications, it is no longer the computer which is central, it is the mass storage which is the central element of the system. It is expected that in the Navy, as elsewhere, many applications which in the past would have used a timesharing mainframe and terminals, will now be implemented with many workstations or PCs and a storage server all tied together by a LAN. The mainframe may still exist, but its significance will be 
reduced, and it will be the storage which is central, not the processors.

\subsubsection{Mini's and Mainframe Computers}

While the growth in workstations and PCs will come somewhat at the expense of mainframes and minicomputers, the Navy's inventory of both will probably continue to grow. In some cases the mainframe or mini will simply serve as the storage element for the workstations. In other cases some or much of the processing function will be retained in the mainframe. Many applications have need for centralized processing and control or are simply not suited to distributed processing. Time sharing is becoming less important, but will not go away. Some applications require more processing power than can be provided by workstations. Many big applications are best addressed as batch applications on big computers. These mainframes and minis will need to communicate with workstations, storage servers and each other. They will do so locally primarily over high data rate LAN networks, and through ISDN to reach remote locations.

\section{3 storage Servers}

The canonical computer system of the past three decades has been a central computer to which a number of storage and I/O peripherals were attached. The paradigm for the next few decades will be quite different. The typical system will have computers scattered wherever people work, and connected together to each other and to mass storage by a LAN. It will be the network and storage, not computers, which are central. The world of computing will, in a sense, be turned inside out. This is because of the development of inexpensive yet powerful microprocessor and high capacity semiconductor memory devices. Where processors were once expensive, they are now very

inexpensive. The appetite of these many processors for mass storage will be insatiable.

Economies of scale apply to electromechanical mass storage devices. Many processors can often share one copy of data or a program, rather than replicate copies at each processor. Many gigabytes of storage costs less in a few large capacity drives than it does in many low capacity drives. A backup device can easily be afforded for a large storage facility. Most important, however, is that central storage facilitates 
management and control of data, while distributing it willy-nilly in many autonomous computers does not. It is difficult to ensure coherence of many independent copies of the same data.

Storage servers of many kinds are the result. Some are simply mainframe computers, which may also supply considerable processing power. Some are simple mass storage servers, which simply replace the local storage with a shared facility: others are data base processors, capable of implementing data structures and performing complex queries. To meet the needs of the processors, very high bandwidth networks will be required. For example, one vendor of diskless workstations states that no more than 6 workstations can be satisfactorily served through one $10 \mathrm{Mbit} / \mathrm{s}$ IEEE 802.3 network. The transfer rate of the slowest commercially available hard disk drives is $5 \mathrm{Mbits} / \mathrm{s}$, and even high capacity 5.25 in drives are typically $10 \mathrm{Mbit} / \mathrm{s}$, with 14 in. drives going up to $24 \mathrm{Mbit} / \mathrm{s}$. The next generation of drives will be faster still. Placing some local hard disk storage at the workstation will sometimes be needed to reduce the load on the network.

\subsubsection{Image Processing}

The expanding interest in image processing applications will generate huge volumes of data to be stored and moved over the network. The following data illustrates the increased data requirements for newer PC and workstation displays:

Display

IBM PC CGA

IBM PC EGA

IBM $P C / 2$ VGA

Macintosh II

IBM PC/2 8514/A

Laser Printer

High Res. Col. CRT

offset Printer
Image

$320 \times 200 \times 2$
$640 \times 350 \times 4$
$640 \times 480 \times 4$
$640 \times 480 \times 4$
$1024 \times 768 \times 8$
$300 \times 300 \times 8 \times 10$
$2048 \times 1024 \times 16$
$1000 \times 1000 \times 8 \times 10$

Mbits per Image

1.229

1.229

6.291

7.200

33.554

80.000

Many future applications contemplate replacing paper copies of documents such as engineering drawings, parts explosions, maps and photographs or maintenance manuals with digitized copies stored on either magnetic or optical storage media. Even allowing for data compression in storage and transmission, this will cause a quantum increase in storage and data transmission requirements in the Navy and elsewhere. 


\subsection{Video}

Video is a vexing subject in this study. Current video standards, such as NTSC, are more than 30 years old. clearly the technology exists to provide much higher quality video than that provided by NTSC. Nevertheless, it is hard to envision that the huge present investment in NTSC compatible video equipment will be largely abandoned in the next decade for some new technology, such as high resolution digital video. The conclusion is, therefore, that the cost effective solution for the remainder of the century to mainstream video needs in the Navy as elsewhere is found in existing analog coaxial cable technology.

Networks

\subsection{Local Area Networks (LANs)}

Local Area Networks are networks which span the area of a large building, a campus or a navy base. If well designed, they need not add appreciably to the mechanical latencies inherent in mass storage servers such as magnetic and optical disk drives, and can have the necessary bandwidth to connect mass storage devices to computers. Thus they facilitate distributed processing and the sharing of mass storage.

\subsubsection{Topologies}

LANS are generally wired as stars, rings or buses. In star networks all stations are wired directly to some central point. Rings are wired in a closed loop. Buses have a linear, sometimes branching structure with taps for stations.

Bus and ring cabling sometimes have the advantage of requiring less cable than star wiring. This may be a particular advantage when adding new cables to an existing facility. On the other hand, star wiring is much the most flexible alternative and offers important maintenance and reliability advantages. Nearly all protocols, whether intended for rings or buses, can be accommodated on star wired cable plants. Rings can in fact be star wired. The broadcast protocols used with buses also can be made to work with star wired cable plants.

With star wiring, there is no need to commit to the precise network configuration at the time a building is wired. Rather, cables are simply run from each room 
back to wiring closets, and those closets are connected to a building master wire room by trunk cables. There is complete flexibility to patch these cables together later at the wire closets as needed.

Star wiring also has important maintenance and reliability advantages. Unlike linear bus cable networks, damage to a cable usually affects only one station. The wire closet provides a central access point to one end of every link for maintenance purposes, avoiding the "pocket full of keys" problem for maintenance of buses (that is, one needs a pocket full of keys to get at the malfunctioning equipment).

For these reasons, telephone service has generally been star wired to wire closets. While some services will be ring or bus wired, the core IAN cable plant proposed for Navy bases is star wired.

\subsubsection{Access Methods}

LANS are also frequently classified in terms of their medium access methods. The three standard methods in common use are Carrier sense Multiple Access with Collision Detection (CSMA-CD), Token Ring and Token Bus. CSMA-CD, probably the most widely used, is a "listen before talk" protocol, which requires the ability to sense packet collisions. It has minimum packet length and maximum network length limitations, and its performance deteriorates under heavy loads due to collisions. It performs very well under light loads. The token ring protocol is well suited to bursty computer traffic and performance does not deteriorate due to collisions under heavy loads. The token bus access method is really not particularly a bus protocol at all; it is suitable for any broadcast medium, bus or star. Its main disadvantage is its complexity and somewhat lesser performance than token ring protocols. It is the perception of the authors of this study that the Token Bus technology is not prospering in the marketplace and it is doubtful that it will be a major factor for the Navy.

\section{1 .3 standards}

While there are many proprietary networks available today, the trend is clearly the use of standard networks to permit multivendor networks. Three network standards, IEEE 802.3, IEEE 802.5 and FDDI are particularly important. 
The first LAN standard to be widely implemented was IEEE 802.3, defining a $10 \mathrm{Mbit} / \mathrm{s}$ CSMA-CD network, using baseband coaxial cable. It is now implemented in products from many different vendors, and is particularly widely used in workstation applications. Digital Equipment Corporation has played a pivotal role in the acceptance and use of this standard. There are now efforts to develop a standard active star fiber based variant, and several vendors now offer fiber based 802.3 variants. Three physical interfaces are really defined in 802.3 : (1) a 0.5 in. coaxial cable trunk bus, which can be tapped by active taps and can run up to 500 meters per segment, (2) a "thinwire" coaxial cable allowing up to about 30 passive "T" taps over a cable up to $185 \mathrm{~m}$ long, and (3) a twisted pair "transceiver interface" using a 15 position connector to connect 2 devices separated by up to $50 \mathrm{~m}$. The maximum end-to-end distance, limited by the collision detection protocol, is nominally $2.5 \mathrm{~km}$.

IEEE 802.5 defines 4 and $16 \mathrm{Mbit} / \mathrm{s}$ token rings which are just coming into wide use today. Since it is the main LAN entry of IBM Corp., it may reasonably be expected to be an important standard. Although, to date, there are comparatively few vendors offering products, it is an open standard. Texas Instruments is selling chips which implement the protocol, and many vendors seem sure to follow. Although it is a ring, 802.5 is explicitly star wired, and relies on using a DC current in the data cable to power a switch in a concentrator to switch stations into the network. Thus, when a station is turned off it is automatically bypassed at the concentrator.

The Fiber Distributed Data Interface (FDDI) is a fiber optic $100 \mathrm{Mbit} / \mathrm{s}$ token ring network. While it was originally developed to serve the needs of mainframe to mainframe and mainframe to storage server applications, its first big commercial uses are apparently turning out to be as backbone networks for other LANs, and as a high performance workstation to storage server networks. At this time, the standard is still not complete and only one company (Fibronics) is shipping products. A number of major vendors are committed to FDDI, however, the most important of which is Digital Equipment Corp., which has launched a major development effort.

A follow on effort is called FDDI-II. It will integrate switched isochronous services with the packet data service and will be suitable for interconnecting 
distributed PBXs as well as computers, storage servers and workstations.

\subsection{Wide Area Networks}

The Navy will use a variety of wide area networks, including private time sharing networks and the Defense Data Network. It is expected, however, that the primary wide area data networks used by the Navy will be based upon the ISDN compatible offerings of the various regional and long distance telephone carriers.

4. Media

\subsection{Electrical Media}

\subsubsection{Twisted Pair}

Twisted pair cable is universally used for voice applications in buildings, between buildings, and over much longer distances in the telephone network. The twists of a twisted pair give this type of cable a degree of protection against interference and crosstalk that is adequate for voice circuits over a distance of many miles. The availability and economy of twisted pair cable has made it the medium of choice for many data applications also. Drops to terminals operating at $64 \mathrm{kbit} / \mathrm{s}$ or less use twisted pair quite routinely. Recent proposals for local area nets suggest that twisted pair may be adequate for much higher data rates, on the order of 1 to $10 \mathrm{Mbit} / \mathrm{s}$. The special problems of transmitting on twisted pair at these data rates will be examined more closely in a later section.

The first issue to be considered in the choice of twisted pair cable is shielding. Shielding usually consists of a metallic foil wrapped around an individual pair and/or a braided wire shield around all the pairs collectively: Shielded pairs have the disadvantage of being considerably more expensive, plus the larger outer diameter of shielded cable makes it much harder to pull through crowded wiring spaces.

Conductor size also affects the transmission capabilities of the cable. Standard telephone cable uses No. 24 American Wire Gage (AWG) wire. Other popular sizes are 22, 26, and 28 AWG. The lower the AWG number, the larger the wire diameter and therefore less electrical resistance. closely related to conductor size is the 
characteristic impedance, zo, of the pair. For a parallel wire line $\mathrm{ZO}$ is primarily a function of conductor diameters and the spacing between them. This relationship is also true for twisted pair but the number of twists per unit length is another variable. Adding a shield around the pair changes the impedance also because of the distributed capacitance between the wires and the grounded shield.

Twisted pair cabling is a very mature technology with a large variety of cable sizes available. Cables with 2 to 600 pairs of unshielded 24 AWG wire are common; with smaller wire, cables with more than 4000 pair can be obtained. Hardware for termination and administration, such as plugs, cross-connects, etc., are also well developed and inexpensive.

Unshielded-pair cable has been the choice for most voice and low-speed data circuits up to now and a substantial number of experts believe that it still has a place in local area networks. One vendor claims, for example, "error free" transmission to 1000 feet at a data rate of $6.3 \mathrm{Mbit} / \mathrm{s}$. Another claims that reliable data transmission requires shielding for any substantial length to protect the data from electromagnetic interference (EMI). As a compromise, this latter vendor offers shielded pairs for data and unshielded pairs for voice in the same cable.

\subsubsection{Coaxial Cable}

Coaxial cable consists of a central wire conductor surrounded by an outer tubular conductor that serves both as a shield and a return path for the signal. The two conductors are separated by an insulating material called the dielectric core. The inner conductor may be stranded or solid; the shield is usually braided strands of very fine wire. In addition, the cable is usually covered with a protective jacket of insulating material.

The characteristic impedance, 20 , of coaxial cable is directly related to the diameters of the inner and outer conductors, and inversely related to the dielectric constant of the core material. Attenuation, loss of signal power, occurs in the conductor, the dielectric, and in radiation from the cable. Attenuation is very much a function of signal frequency. Though there are many factors in coax cable design that affect attenuation, it can be generally stated that larger diameter cables have less attenuation at high fre- 
quencies than small cables. For example, MIL-Spec RGIIA/U, with a diameter of $0.7 \mathrm{~cm}$ has an attenuation of $75 \mathrm{~dB} / \mathrm{km}$ at $100 \mathrm{MHz}$; RG319/U has an attenuation of only $7.2 \mathrm{~dB} / \mathrm{km}$ at that frequency but its diameter is $4.1 \mathrm{~cm}$.

Coaxial cable has been used for many years in broadband analog applications such as CATV distribution, and, to a lesser extent, for data networks. It has an advantage over twisted pair in terms of signal bandwidth but a disadvantage in terms of cost and physical flexibility. The most effective use of coax is in bus topologies where many users share the same cable by means of taps that connect terminals to the main spine cable. The physical bulk of coax and the greater difficulty of installing connectors make it less attractive for applications that require massive numbers of cross-connects or patch panels.

With care and proper planning, it is possible to have coaxial cable and twisted pair in the same signal path. This may be desirable for example when a data device designed for a coax interface needs to be connected and only twisted pair cable has been installed. The important electrical difference is that twisted pair transmission is usually a balanced line, i.e., neither conductor is grounded; at the transmitter and receiver the impedance from each line to ground is equal. Coaxial lines, on the other hand, are inherently unbalanced since one conductor (the shield) is always grounded. To connect the two types together a device called a balun (for balanced-unbalanced) is used. This is basically a small transformer with a jack for coax on one side and a twisted pair connector on the other. Though baluns are relatively inexpensive and easy to use they should not be installed without due consideration for the effect on the circuit. The balun itself will add some attenuation and the maximum allowable length for the combined line will almost certainly be different than for either twisted pair or coax alone.

\subsection{Optical Media}

optical fiber waveguide offers several advantages over all types of copper media, namely greater bandwidth, less attenuation, smaller cable cross section, total electrical isolation, and freedom from interference and crosstalk. Fibers are not susceptible to electromagnetic interference nor do they cause spurious emissions. The available bandwidth of fiber relative to its size is perhaps the most impressive advantage; in terms of raw bandwidth a pair of 
fibers in a 0.25 inch cable can carry as much information as several hundred twisted pair.

An optical fiber is a circular dielectric waveguide, usually silica glass, consisting of an inner core surrounded by a concentric cladding of a slightly lower refractive index. Superficially, the guiding of light in the core can be explained by ray optics and total reflection at the corecladding boundary, but more precise descriptions require the use of guided wave theory. The fiber geometry and composition determine a discrete set of electromagnetic fields which can propagate in a fiber. The term "mode" is used to refer to a single electromagnetic field distribution that satisfies Maxwell's equations and the boundary conditions given by the fiber. Loosely speaking, each mode may be thought of as a separate path along which light may pass through the fiber. There are two broad classifications of fiber construction, multimode and single mode.

\subsubsection{Multimode Fiber}

Fibers constructed with a geometry that allows many modes to propagate are called multimode fibers. There are two distinct types, step index and graded index. A step index fiber has a core with a uniform refractive index. Each mode that propagates in the core has its own distinct velocity because each traverses a slightly different path as it goes through the fiber. This means that there is a slightly different arrival time at the receiving end, which causes a signal distortion called modal dispersion. In graded index fiber the refractive index is varied across the core so as to develop a parabolic profile as a function of radius. With this structure, the rays nearest the axis travel through the region of highest refractive index and thus, traveling more slowly, arrive at the same time as the rays travelling a longer path. Graded index fibers thus have less modal dispersion and have a higher bandwidth than step index fibers.

One measure of the fiber's ability to gather light from a source is its numerical aperture (NA). The NA is defined as the sine of the largest angle that an incident ray can have for total internal reflectance. The NA figure is a function of the difference in refractive indices of core and cladding. The NA is one qualitative indicator for how easy it is to couple light into a fiber. For coupling light, the higher the NA the better, but there are certain tradeoffs. For example, increasing NA by making the core index higher causes higher loss in the fiber. 
Core and cladding diameters are also relevant to the performance of the fiber. These diameters are usually expressed in micrometers, e.g., 50/125, for core and cladding respectively. The four popular multimode sizes are 50/125, 62.5/125, 85/125, and 100/140. Increasing the core size increases the source coupling efficiency and lowers the losses in connectors but it also increases certain losses in the fiber and decreases the bandwidth of the fiber. The 50/125 size is popular for long haul communications; $62.5 / 125$ is preferred for local networks. A cladding size of 125 micrometers is desirable because of the wide availability of connector and splice hardware for this size.

Proposed Federal Standard 1070 specifies $62.5 / 125$ graded index fiber for IAN and PBX-type applications in the Federal Government. This fiber is already becoming the de facto standard for these applications in the United states. This is the only fiber type ordinarily required to cable Navy bases.

\subsubsection{Single Mode Fibers}

A single mode fiber has a step index profile with a core size so small that only a single mode will be propagated at the operating wavelength. Since there is only one mode, modal dispersion ceases to exist, giving this type of fiber an extremely high bandwidth. The small core diameter causes some problems, however, making it difficult to launch enough light from a source and making the job of splicing and connectorizing very challenging. For long distance communications this is a small enough price to pay; for long hauls the source is usually a more expensive laser diode rather than an LED and the links are not connector intensive. Moreover, expensive equipment and specialized procedures for handling of the fibers add only a small increment to the cost of long links and are more than offset by the savings realized by needing fewer repeaters.

At the present time, multimode fiber seems more than capable of meeting the Navy's voice and data transmission needs within the confines of most bases

at a much lower cost than if single mode were used. It is hard to name a specific use for single mode fibers in Navy base applications today, where a similar service could not be provided at lower cost using multimode fiber. Perhaps the first application where single mode transmission will prove to have a clear advantage will be analog video distribution. None the 
less there is a persistent suspicion that this may change and it seems prudent in some circumstances, such as where streets must be dug to lay cable, that some single mode fiber should be included, even though it may lie dark for many years before it finds a use.

5. Commercial Wiring Plans

In recognition of the multitude of problems associated with building wiring obsolescence, several large firms have announced proprietary premises wiring systems in the past few years. The important common feature of these systems is that they offer a well developed architecture and strategy for wiring as well as a set of wiring components, planning guides, and consultative services. Three major sources, IBM, AT\&T and DEC, are summarized below.

\subsection{IBM Cabling system}

The IBM Cabling System was announced in 1983 and made available in late 1984. It is based on shielded 22 AWG twisted pair copper cable for both between closet and closet to wall outlet wiring. Optical fiber or twisted pair can be used for backbone wiring. The cabling system is consistent with that described in IBM's contributions to the local area network standards committees of the IEEE 802.5 and ECMA 89. These contributions describe a star-wired, token ring LAN and its relationship to IBM's systems Network Architecture (SNA). Highlights of the IBM plan:

* Supports IBM Token Ring. This is a $4 \mathrm{Mbit} / \mathrm{s}$ network that conforms to IEEE 802.5 .

* Topology is a physical star, logical ring. star wiring connects work stations to a wiring closet where logical ring control is handled by a central control device. A DC current supplied by stations is used to close a relay which switches the station into the ring. Controllers are available for both copper and optical fiber.

* Whether or not a ring topology is used, cables from work areas all terminate at a distribution panel in a wiring closet. The function of these is much the same as that of a traditional telephone wiring closet or the familiar computer room patch panel.

* Basic cable (Type 1) is two shielded twisted pair, 22 AWG. Type 2 cable has this plus 4 unshielded pair for telephony. Type 5 is fiber optic cable with two $100 / 140$ fibers that can be used for the backbone (riser) subsystem, indoors or out. 
* In the work area, the cables terminate in wall jacks (or floor pedestals) that accept a standardized data plug or a modular phone plug.

* Special cable assemblies containing baluns are used to convert coax, twinax, financial loop, and other products to the twisted pair medium.

In addition to the Types 1 and 2 shielded cables, a few other twisted pair cables are allowed for special applications:

Type 3. Unshielded twisted pair, four pair per cable, may be 22 or 24 AWG, (not manufactured by IBM). For low speed data and telephony.

Type 6. Two twisted pairs of 26 AWG. For making patch cords to be used in the wiring closets.

Type 8. Two parallel pairs of 26 AWG in a flat configuration for under-carpet installation.

Coaxial cable is allowed for specific device connections but not as a part of the building wiring scheme.

Maximum cable runs are given in terms of equivalent length of Type 1 cable. To be guaranteed compatible with the token ring LAN, the absolute maximum cable length between wiring closets is 770 meters and the maximum drop length (closet to wall outlet) is 100 meters. Using cable types other than Type 1 or 2 reduces the distance allowed by a factor of 1.5 to 2. Adding items such as surge supressors or baluns subtracts from the maximum figure also. Fiber optic cable length between closets can be 2000 meters; fiber is not used for drops.

\subsection{AT\&T Premises Distribution System (PDS)}

Based on unshielded twisted pair for distribution of both voice and data, the AT\&T system offers a common distribution architecture for tying together their voice and data products as well as those of other vendors. The rationale of their plan relies heavily on the economy and convenience of using the twisted pair technology that is mature and is already installed in a large number of buildings. Furthermore, the plan promises easy voice and data integration possibilities with support of PBX's and eventual migration to ISDN. 
AT\&T aims to be strongly committed to the use of optical fiber and has included fiber cable and optical multiplexers in their plan to implement certain backbone functions. As with the IBM system, coaxial cable is permitted for connection to specific devices where needed but is not allowed to be part of the installed building wiring. Other major features of the PDS:

* Supports AT\&T starlan 1Mb/s CSMA/CD LAN, $8.64 \mathrm{Mbit} / \mathrm{s}$ ISDN baseband network, and system 85 and 75 PBX's.

* Hardware is available to convert WANGnet and IBM 3270 equipment (coax based) to the PDS twisted pair.

* Topology is modified star with workstation outlets connected to satellite closets which are then connected to common equipment and/or computer rooms. Backbone (riser) subsystem connects closets to main administration points. An administration point ties other subsystems together and permits routing; provides both copper and fiber interconnects and cross-connects.

* Typical closet-to-wall run uses two 4-pair cables consisting of 24 AWG wire. The cables are each terminated in an 8-pin modular jack. Shared jack arrangements are possible, e.g., an IBM terminal and an analog telephone on one jack and one 4-pair cable run.

* Fiber optic cable is used extensively in the backbone. Recommended fiber size is 62.5/125. A ribbon design cable is available which can contain up to 144 fibers. Ribbons are encased in a round sheath.

Recommended maximum signal path lengths vary according to the anticipated application. Maximum distances served are stated in the specifications for each PDS product. Some examples:

* Copper to fiber adapter, 32 ports for IBM 3270 terminals. Distances to $5000 \mathrm{ft}(1515 \mathrm{~m})$.

* Coax to twisted pair adapter for WANGnet, $700 \mathrm{ft}$ (212 m) at $4.275 \mathrm{Mbit} / \mathrm{s}$.

* Asynchronous RS-232C terminals on twisted pair, $20,000 \mathrm{ft}(6 \mathrm{~km})$ at $1.2 \mathrm{kbit} / \mathrm{s}$.

* Maximum length of fiber backbone is typically 9600 ft $(2.91 \mathrm{~km})$.

* Twisted pair cable with 24 AWG wire is rated error free to $1000 \mathrm{ft}(303 \mathrm{~m})$ at $6.3 \mathrm{Mbit} / \mathrm{s}$. 
AT\&T also offers services with their plan, such as site survey, distribution planning, product procurement, installation and maintenance. The plan manuals cover most of these topics in a general way but they also stress that company representatives may be consulted for individual cases.

\subsection{DECconnect}

In contrast to the preceding plans, in which coaxial cable is all but eliminated as an approved transmission media, Digital Equipment Corporation (DEC) uses the coax-based Ethernet IAN as the centerpiece of its DECconnect wiring plan. Starting with hosts connected to the "standard Ethernet" 0.5 inch coax for the backbone of the system, DECconnect extends the data path to wall outlets in offices with a lighter and more flexible cable called Thinwire coax. Both cables support the Ethernet $10 \mathrm{Mbit} / \mathrm{s}$ baseband data rate. Fiber optic cable is included in the plan, primarily to connect Ethernet LANs located in separate buildings or for specialized connections within a building. Twisted pair has its place too, as the distribution medium for low speed data and voice.

The single point of contact between users and all communications networks in the facility is the DECconnect faceplate located on a wall or in a floor pedestal. The faceplate contains four receptacles at one outlet box to support the following functions:

* A $10 \mathrm{Mbit} / \mathrm{s}$ local area network (Ethernet) on Thinwire coax BNC connector.

* A modified modular jack for connecting non-intelligent terminals and devices such as printers using unshielded twisted pair. (Data rates to 19.2 $\mathrm{kbit} / \mathrm{s}$ )

* A modular jack for connecting telephone equipment to the voice network.

* An F-connector for connections to the CATV network.

Cables from all of the above are concentrated at wiring closets which they call satellite equipment rooms (SER'S). The twisted pairs are terminated in patch panels in the SER; those requiring network connection are patched through concentrators to a terminal server which multiplexes these lines to a single Ethernet connection. 
Thinwire cables from the faceplate are patched through to an Ethernet multiport repeater (DEMPR); each repeater can handle eight connections. The repeater retimes and amplifies all signals received on a Thinwire segment and retransmits them to the rest of the network. One segment can be up to 185 meters long.

A local network interconnect (DELNI) is next in the hierarchy of concentrators. It can connect eight repeaters or eight terminal servers (or a combination of the two) to the backbone network via an Ethernet transceiver. The transceiver attaches to a standard size cable with a non-intrusive tapping device, i.e., the cable need not be cut. Units can be installed or removed without network interruption.

From the SER, Standard Ethernet cable connects to other SERS or to a host computer in the main equipment room. A stand-alone standard Ethernet segment can be up to 500 meters long, but when used with the concept described above using repeaters and interconnects the distance is limited to 300 meters because of the added propagation delays in the electronics.

Extensions of the network over wider areas is done by using bridges. The bridge performs traffic management functions that keep the network from being overwhelmed by the combined traffic of multiple networks. The LAN Bridge 100 model is used when buildings are up to $2 \mathrm{~km}$ apart on a campus. The transmission medium between bridge units is usually optical fiber. Up to seven bridges may be added together serially to form very long links or bridges can be placed in parallel to increase the availability and reliability of the network.

Recent Developments in Wiring Technology

6.I Unshielded Twisted Pair for IEEE 802.3 Applications

Using conventional telephone wires for local area networking is undeniably appealing for reasons of economy and convenience. Not only is the twisted copper cable the least expensive media but it offers many other advantages, mostly due to the many years of development that twisted pair technology has been through. Nearly all buildings built today, whether for offices or other purposes, have wiring closets and distribution ducts designed for the star topology used by the telephone system. The wiring closet is the hub of the star and is an ideal place for punch-down blocks, patch panels, concentrators, and such hardware that allows reconfiguration, modification, and troubleshooting with a minimum of disturbance to the wiring plant. It is a 
natural extension then, when going from voice only systems to voice and data, to use these wiring centers as a place to install repeaters and other active network components.

Furthermore, a data network based on a ring or bus topology can utilize the star wiring scheme quite easily by using twisted pair wires for individual drops to the closets and a higher bandwidth medium for inter-closet trunks.

In the past year much attention has been focused on twisted pair implementations of the 10 Mbps 802.3 networks.

Conventional wisdom holds that $10 \mathrm{Mbps}$ over voice grade

lines is very perilous because of a) high attenuation, b) rf emissions, c) susceptibility to electromagnetic interference (EMI), and d) crosstalk. These four issues have been examined in detail by vendors of 802.3 equipment and some promising solutions have been put forth.

For purposes of the following discussion, conventional telephone wiring is assumed to be unshielded copper pair with 24 AWG conductors, also known as D-inside wire (DIW). Usually four or more pairs are put into one cable; if limited to four the cable is quite small and flexible. The characteristic impedance of DIW is about 100 ohms and the attenuation at $10 \mathrm{MHz}$ about $3 \mathrm{~dB}$ per 100 feet.

Attenuation obviously places a restriction on the maximum length of cable; the difference between transmitted power and receiver threshold yields a power budget figure from which this length can be calculated. This figure, however, should not be used as the actual useful length; there are other issues related to length that must be considered, such as crosstalk, jitter, and receiver input design. Crosstalk between pairs can be measured by attaching a transmitter to one pair and a receiver to an adjacent pair at the same end. This will yield a figure for near end crosstalk which has been found to be less than $-22 \mathrm{~dB}$ at 10 Mbps. [Ref. 10] If the receiver in the system is to ignore crosstalk its threshold must be set above this level.

The relationship between attenuation and jitter is due to the fact that attenuation is frequency dependent; high frequency components of the signal are attenuated more than low frequency components. In the Manchester encoded data used in 802.3 , the frequency components of a given bit depend on the data immediately preceding, so the frequency content is data dependent. The effect of variable attenuation then is to cause deviations of the data transitions from the center of the bit cell. The 802.3 specification allows no more than plus or minus 13 nanoseconds deviation. A twisted pair system can be made to stay within this limit by incorporating an equalizer into the receiver. The equalizer is a circuit that amplifies the 
high frequency components more than the low frequencies, thereby compensating for the frequency-dependent attenuation.

High frequency signals on unshielded conductors will radiate energy into the local environment; this can cause serious problems, particularly interference with broadcast communications signals. The FCC has regulations limiting emissions above $30 \mathrm{MHz}$. Since the primary component of the 802.3 signal is $10 \mathrm{MHz}$, it is possible to limit all the higher frequency components with filters and still maintain the integrity of the data.

Unshielded cable is also a good receiving antenna for picking up interference from a variety of sources, so a system must be designed to reject such interference. The key to such design is the fact that twisted pair is a balanced transmission line; this means that differential drivers and receivers can be used. The differential driver puts signals on the two wires that are 180 degrees out of phase with each other; the receiver is designed to accept only the signals that are out of phase and reject all others. The interference signals are generally in-phase since the noise is picked up equally by both wires. This is called common mode noise. The common mode noise can be large enough to overload the receiver, so in addition to the differential design it is also necessary to protect the input with a common-mode filter. This type of filter allows differential signal to pass but highly attenuates common mode signals. It generally consists of a choke inserted in each line.

\subsection{Standards Committee Work}

The IEEE 802.3 standards committee has a study group called lobase-T which is currently studying the issues of using unshielded twisted pair. After several months of examining different topologies that had been proposed to the group, it appears the group has settled on one which twisted pairs connect transceivers at a wiring hub to transceivers at or near each user's desktop. The wiring hub includes an 802.3 multiport repeater; patch cables from the repeater go to a punchdown block and from there twisted pair runs up to 100 meters to standard modular phone jacks. From the phone jack a patch cord connects to a media access unit which has an interface compatible with 802.3 so that it can connect to the standard driver board in the workstation. With the multiport repeater located in a telephone wiring closet and connected to offices by standard (even existing) telephone cable and hardware, this scheme easily coexists with present voice service and future ISDN integrated services. 
An alternate topology considered by the committee is daisychaining. In this architecture, a multiport repeater connects to twisted pairs through transceivers as above, but the other ends of the pairs do not go to the individual workstations but to a twisted pair-to-coax adapter. From the adapter, thin coax runs to each station in the usual way, with up to 25 stations on one segment. The advantage claimed for daisy-chaining is that it reduces the cost of changing from an existing 802.3 network because the user does not have to replace the transceiver at each station with a twisted pair model. Also, this scheme conserves twisted pairs since only two pairs are needed to network 25 stations. However, daisy-chaining retains many of the disadvantages of wiring with coax, especially those of being difficult to relocate stations and not being compatible with telephone-type wiring. As of this writing, daisy-chaining has been withdrawn from 10Base-T consideration but its proponents continue to offer it as a commercial product.

\subsection{New Wire Types}

The above efforts are aimed at putting 802.3 signals on standard D-inside wiring. There are some recent developments in the manufacture of twisted pair cables which promise to extend the distance of data transmission significantly. Dupont claims that wires insulated with a fluorocarbon resin instead of the usual PVC reduces the capacitance of the pair by about 50 per cent. PVC cables have a capacitance range of 25 to 35 picofarads ( $\mathrm{pF}$ ) per foot; with fluorocarbon insulation the reported range is 12 to $18 \mathrm{pF}$ per foot. Another company, NEK Cables, claims they are achieving capacitance as low as $8.5 \mathrm{pF}$ per foot by "foaming" the cable (adding air bubbles to the insulation). [Ref. 11] Since cable capacitance is a major factor in signal degradation, these reductions are said to increase the maximum distances proportionally. An added advantage to the fluorocarbon insulation is that it is teflon coated so that it can be pulled through plenum areas without the need for conduit. The foamed insulation reduces the weight of the cable and gives it increased flexibility during installation.

\section{Conclusion}

No wire plan for Navy bases can provide for every possible future need. In particular, there exist numerous LAN networks with rather different cabling requirements. Never the less, it is possible to establish a straightforward approach to wiring Navy 
bases, which will handle the vast majority of voice and data applications. In summary this approach is:

* The universal floor wire closet to work station wall outlet connection is two bundles of 4 twisted pairs.

* Multimode fiber, coaxial cable and shielded twisted pair cables to wall outlets may be required in specific cases, used only on an "as needed" basis.

* Intra-building trunks connecting floor wire closets and the building wire center will be a mix of twisted pair and fiber cables.

* Inter-building trunks connecting building wire centers and the Base wire Center will be multimode fiber.

This approach differs from conventional practice only in that the use of coaxial cable is minimized for trunks, while multimode fiber is emphasized. The greatest uncertainty at this point is the desirability of routinely adding single mode fiber to interbuilding trunks. There is no immediate general use for such fiber, but at some time in the next decade broadband ISDN services may be offered to users; these will use single mode fiber. Another possible use for single mode fiber is in the future distribution of high resolution video.

It is difficult to predict which proposed new video services may be offered commercially in the reasonably near future, or the pace at which new video services may develop. Under these circumstances, conventional coaxial cable based video distribution systems appear to be the prudent choice.

\section{REFERENCES}

1. Draft Proposed American National Standard, Fiber Distributed Data Interface (FDDI), Token Ring Physical Layer Medium Dependent (PMD), X3.166-198X.

2. American National Standard, Fiber Distributed Data Interface (FDDI) Token Ring Media Access Control (MAC), X3.139-1987.

3. American National Standard, Fiber Distributed Data Interface (FDDI), Token Ring Physical Layer Protocol (PHY), X3.148-1988.

4. Draft Proposed American National Standard, Fiber Distributed Data Interface (FDDI), Token Ring Station Management (SMT), X3T9.5/84-49 Rev. 5.0, August 1988 .

5. Token Ring Access Method and Physical Layer Specifications, ANSI/IEEE Standard 802.5-1985. 
6. Carrier Sense Multiple Access with Collision Detection (CSMA/CD), ANSI/IEEE Standard 802.3-1985.

7. Draft Standard of ISDN Basic Access Interface for Application at the Network Side of the NT Layer 1 specification, ANSI/TID1.3/87-003R4, May 221987.

8. Detail Specification for 62.5 um Core Diameter/125 um Cladding Diameter, Class Ia Multimode, Graded-Index optical Waveguide Fibers, EIA-492-AAAA, 1988.

9. Hull, J.A., and Hanson, A.G., "On-Premises Digital Communications Upgrades With Emphasis on Fiber Optics," NTIA Special Publication 86-18.

10. Schmidt, R.V., "Developing Ethernet Capability on Unshielded Twisted Pair", Telecommunications Magazine, January 1988.

11. Frost \& Sullivan Report No. A1772, "Premises Distribution Systems in the U.S."

12. AT\&T, Premises Distribution, Introduction, Doc. \# 999-300$1961 S$.

13. AT\&T, The AT\&T Premises Distribution System, Doc. \# 555-400022 .

14. AT\&T, Premises Distribution, Guide, Doc. \# 555-400-021.

15. DEC, The DECconnect Communications System Handbook, Pub. \# EB-28987-42.

16. DEC, DECconnect system Planning and Configuration Guide, Pub. \# EK-DECSY-CG-001.

17. DEC, DECconnect System Installation and Verification Guide, Pub. \# EK-DECSY-VG-001.

18. IBM, A Building Planning Guide for Communication Wiring, \# G320-8059-1.

19. IBM, IBM Cabling System Planning and Installation Guide, \# GA27-3361-6

20. IBM, Using the IBM Cabling System with Communication Products, \# GA27-3620-1

21. Draft Commercial Building Wiring standard, EIA TR-41.8.1, Draft 3.0, April 1988. 


\title{
APPENDIX A
}

\author{
D R A F T
}

PROPOSED

\section{NAVAL DATA AUTOMATION \\ - TECHNICAL STANDARD}

\author{
PREMISES WIRING PLAN \\ FOR \\ DATA, VOICE AND VIDEO
}

August 1988 
DRAFT

TABLE OF CONTENTS

\section{Page}

PARAGRAPH 1 .

AUTHORITY

1

2 .

POLICY

1

3.

PURPOSE

1

4.

APPLICABILITY AND SCOPE

1

5.

INFORMATION AND ASSISTANCE 3

6.

REFERENCES

3

7.

SUPPLEMENTARY MATERIALS

5

8.

PROCEDURES

5

8.1

Base wiring Center

8.2

Inter-Building Trunks

8.3

Building Master Wire Center

5

8.4

Intra-Building Trunks

6

8.5

Secondary wiring closets

9

8.6

Closet to Work Station Cabling

8.7

Work Station outlet

13

8.8

Documentation and Labeling

14

17

8.9

Administration and Management

18

21

9.

IMPLEMENTATION

21

10.

RESPONSIBILITY

23

11.

SPECIAL INSTRUCTIONS AND COMMENTS 23

ATTACHMENT I.

Wiring for Modular Jack Receptacle I-I 


\section{DRAFT}

SUBJECT: PREMISES WIRING PLAN FOR DATA, VOICE AND VIDEO

1. AUTHORITY. Navy-wide information systems (IS) technical standards are issued under the authority of OPNAVIST 5450.200, Subject: Naval Data Automation Command; mission and functions of, and SECNAVINST 5230.8, Subject: Information Processing standards for computers (IPSC) Program. The publication of this standard is consistent with the overall Naval Data Automation Command (NAVDAC) mission, functions, and IPSC Program responsibilities.

2. POLICY. To develop and use IS technical standards that contribute to the economic and efficient use of Is resources, and to avoid the proliferation of local or vendor-unique standards.

3. PURPOSE. This document proposes a common approach to wiring Navy bases for voice, data and video. Having a structured cabling plan will simplify installation of new equipment, expedite relocation of existing equipment and reduce connectivity problems between different kinds of equipment.

4. APPLICABILITY AND SCOPE. This plan is intended primarily for use in new construction or in situations where major renovation PAGE 1 OF 25 PAGES 
DRAFT

of existing facilities calls for fundamental redesign of building wiring. As a further guide to applicability, it should be noted that certain assumptions were made in drafting this document regarding the communications and data processing environment. These assumptions are as follows:

* While many kinds of LANs will be found throughout the Navy, the ones which will be widespread are IEEE 802.5 (IBM Token Ring), IEEE 802.3 (Ethernet) and Fiber Distributed Data Interface (FDDI).

* Widespread terminals will be conventional asynchronous terminals up to 19.2 Kbaud, IBM 3270 (SNA) type terminals and new ISDN Integrated Voice/Data terminals operating at 64 kbits/s.

* The principal wide area data network for the Navy, as for the rest of the country, will be ISDN services offered by public telephone companies and long distance carriers.

* Broadband coaxial cable networks will not be widespread, except, perhaps, in manufacturing automation applications.

* NTSC video will not be supplanted in the US in this century, although other high resolution, possibly digital,

PAGE 2 OF 25 PAGES 


\section{DRAFT}

video services may make their initial commercial appearance in this time frame.

* Increasing communications needs and advancing technology cause rapid obsolescence of any wiring system that is designed only to meet current requirements.

5. INFORMATION AND ASSISTANCE. Inquiries and comments on the IS technical standards may be directed to NAVDAC, via:

a. Telephone: AUTOVON: 288-3869

Commercial: (202) 433-3869

b. Correspondence: Commander, Naval Data Automation Command Systems Software, Teleprocessing and Standards Directorate (Code 30) Standards Department Washington Navy Yard Washington, DC 20374

6. REFERENCES. The following documents are pertinent to the development and implementation of this standard. Information on how to obtain these documents is given in section 11 of this document. 
DRAFT

a. Draft Proposed American National Standard, Fiber Distributed Data Interface (FDDI), Token Ring Physical Layer Medium Dependent (PMD), X3.139-198X.

b. American National Standard, Fiber Distributed Data Interface (FDDI), Token Ring Media Access Control (MAC), X3.1391987 .

c. American National Standard, Fiber Distributed Data Interface (FDDI), Token Ring Physical Layer Protocol (PHY), X3. $148-1988$.

d. Draft Proposed American National standard, Fiber Distributed Data Interface (FDDI), Token Ring station Management (SMT), X3T9.5/84-49 Rev. 5.0, August 1988.

e. Draft standard for ISDN Basic Access Interface for Application at the Network side of the NT Layer 1 specification, ANSI/TID1.3/87-003R4, MaY 22, 1987.

f. Detail Specification for 62.5 um Core Diameter/125 um Cladding Diameter Class Ia Multimode, Graded-Index Optical Waveguide Fibers, EIA-492-AAAA, 1988. 


\section{DRAFT}

g. Token Ring Access Method and Physical Layer Specifications, ANSI/IEEE Standard 802.5-1985.

h. Carrier Sense Multiple Access with Collision Detection (CSMA/CD), ANSI/IEEE Standard 802.3-1985.

i. Draft Commercial Building Wiring standard, EIA TR41.8.1, Draft 3.0, April 1988.

j. Draft Building Standards for Telecommunications Media and Systems, EIA TR-41.8.3, Draft 4, May 20, 1988.

\section{SUPPLEMENTARY MATERIALS}

a. Attachments

I. Wiring for Modular Jack Receptacles.

\section{PROCEDURES}

8.1 Base Wire Center. The base wire center is the point of entrance for the public telephone network, the Defense Data Network (DDN), and any other wide area networks whether they come in from cable, microwave, or satellite dish. The center will contain receiving and transmission equipment for the wide area PAGE 5 OF 25 PAGES 


\section{DRAFT}

networks and gateway, bridge, and interface equipment for the local area network(s). For the telephone network, a private branch exchange (PBX) will be located in or very close to the wiring center. The local network will likely require a host computer or master controller to be located in this vicinity also.

The size of the center will be determined after acquiring specific information about the equipment to be installed. The communications equipment will require environmentally controlled space, safe from natural hazards such as floods and secured against intrusion. Physical security is essential, because the destruction of the wire center will paralyze communications on the base. In addition to allowing space for the computers, transmitters, controllers, etc., consideration must be given to some less obvious points. For example, if the PBX selected has battery backup capabilities, a separate room must be provided for the batteries. Cable entrances from above-ground lines require not only termination panels but a place to mount lightning and surge protectors. Special grounding devices and schemes may have to be installed, preferably before the building is constructed.

8.2 Inter-Building Trunks. The inter-building trunks are the communications lines emanating from the central communications PAGE 6 OF 25 PAGES 


\section{DRAFT}

equipment at the base wire center to the wire centers in the individual buildings of the facility. The transmission media will be optical fiber cable to the maximum extent possible. For larger installations, voice, data, and video may share the same fiber by using time division multiplexing transmission techniques; simpler systems may use separate media for baseband data networking and conventional voice circuits.

There are several installation techniques for inter-building trunks, each with its own set of trade-offs in terms of cost, performance, appearance, flexibility, and security. The relative importance of these is usually application dependent and is different for new construction than for site upgrades. The following is a brief discussion of the four most common methods.

* Underground Conduit. Conduit of corrosion resistant material should be buried to a depth of at least 18 inches or whatever depth local codes require. Access to connection points is provided by manholes. Conduit diameter of 4 inches is common, but questions of how big, how many, and where to are site dependent. Properly installed, conduit offers excellent mechanical protection and physical security. Additional cable is installed with relative ease if adequate capacity has been provided in the initial planning. 


\section{DRAFT}

* Direct Burial. Direct burial refers to the technique of laying cables with appropriate sheathing qualities directly in a trench. Usually the trenching, laying of cable, and cover-up is done by one machine in one pass (plowed in). Direct burial is less costly than conduit but offers less protection. A rust resistant metallic armor is often used to protect against damage by rodents. In rocky soil the cable is laid in a layer of sand to prevent puncture of the sheath by sharp rocks.

* Utility Tunnel. Some sites have tunnels between buildings for steam pipes and other utility distributions; these offer a relatively secure and convenient facility for cable installation at minimal additional cost. Cables must be placed far enough away from steam pipes to prevent damage caused by heat or possible steam leaks and high enough to prevent damage caused by flooding.

* Aerial Distribution. Communications cables suspended from poles is generally considered the least desirable method of distribution. It is a very inexpensive method, particularly if the poles are already in place, but offers very little protection from weather, degrades the appearance of the site, and is very vulnerable to damage by motor vehicles, vandals, etc. 


\section{DRAFT}

The above comments on distribution methods apply equally well to all types of transmission media. Cable vendors are able to supply cable for all these types with optical fiber or coax as well as twisted pairs. The choice of media will of course affect the size of conduit to be buried, optical fiber requiring far smaller diameters for equal amounts of information transfer.

When the installation of cable requires special construction and when subsequently adding additional circuits would require repetition of this expense, care should be taken to ensure adequate growth capacity, even if cables may remain unused for many years. This is particularly likely to be the case when cables are buried. In many cases it will be sensible to install 8 or 12 fiber cables where a single pair would handle the immediate need. It may also be prudent in such cases to include some single mode fiber, even though there may be no immediate application for it.

8.3 Building Master wire center. The building master wire center is where inter-building trunk cables interface with the building cable system. The size of the center depends on the type and use of the building. For large office buildings (20,000 


\section{DRAFT}

sq. It and over) the center should be a separate room, rather than a closet or portion of a room, since there will certainly be several racks of equipment to be housed. A rule of thumb is that the room be a minimum of 8 feet wide with $1 \mathrm{sq}$. ft. of floor space for every $2,000 \mathrm{sq}$. ft. of office space. For buildings smaller than $20,000 \mathrm{sq}$. ft. and where the service required is primarily telephone, a large closet or wall space in a secure utility room may be adequate. When wall mounted terminations are used, 3/4 inch plywood should be securely attached to one wall as a place to mount the terminal blocks and cable strain reliefs.

Location of the wire center within the building should be close to the point where the communications cables enter the building and, if possible, near the center of the intra-building distribution system if long horizontal runs are anticipated. For multistory buildings, place the wire center on the lowest level so that it is in vertical alignment with the riser subsystem. Rooms should be dust free, environmentally controlled, have overhead lighting, and be provided with 20 ampere, 115 volt outlets for powering electronic equipment. Doorways should be at least 36 inches wide by 80 inches to accommodate standard equipment racks.

8.4 Intra-Building Trunks. Intra-building trunks are the "backbone" of the local area networks. The backbone subsystem PAGE 10 OF 25 PAGES 


\section{DRAFT}

connects the building master wiring center to the secondary wiring closets or connects closets to each other. In multi-story buildings, the cable routes that bring the backbone from one floor to the next are called "risers." The backbone media is either optical fiber or twisted pair cable, terminated in interconnect panels, multiplexers, "punch-down blocks", splice cases, or concentrators.

Distribution methods for the trunks vary according to building design and construction techniques. In multistory buildings where closets are vertically aligned, the cables may simply go through slots in the floor and ceiling of each closet or through short pieces of conduit called sleeves. Space in elevator shafts may be used for cables, but extra protection and mechanical support may be required.

Conduit is often used where closets are vertically offset or where the distribution is mainly horizontal. The advantages of conduit are that it offers mechanical protection, it is fire resistant, and provides a concealed housing in areas where wiring might otherwise be exposed. The disadvantages are that it requires extensive planning to provide adequate sizes and placement to proper locations and it is difficult to change or add to after the building is finished. 


\section{DRAFT}

For horizontal runs where appearance is not important, cable trays may be used. These are aluminum or steel assemblies resembling ladders that are suspended from ceiling beams. Trays allow easy placement of cables and eliminate the problems of pulling through conduit. Disadvantages are that cable is left exposed, and is therefore difficult to fireproof and to protect from intrusion.

A few rules of thumb for intra-building trunks:

* No conduit run should be more than 200 feet long or contain more than two 90 degree bends unless pull boxes are installed in the run.

* For telephone cable, run 15 pairs per 1000 square feet of office space or 2 pairs per residential unit. It is better to run one cable per secondary closet rather than have one cable serve many closets.

* For conduit sleeves between floors, install two 4 inch sleeves plus one spare, for up to 50,000 square feet of floor space. For additional units of 100,000 square feet provide an additional sleeve. 
DRAFT

8.5 Secondary Wiring Closets. Secondary wiring closets contain patch panels, cross-connects, concentrators, multiplexers (electrical and optical), and any other electronics or adapters needed to convert the backbone signals for distribution to specific devices. Secondary closets contain some of the same equipment as the building wiring center, but they supplement the wiring center to permit extension of the network over greater distances, or to support more workstations than one center could reasonably handle.

Design considerations for secondary closets are based on the same criteria as is the building wiring center but the scale is usually smaller. The prime considerations are the size of the communications system and the square footage of office space to be served. Location decisions depend on the maximum allowable drop length for specific subsystems and best access to the intra-building trunk.

The secondary closet must be a lockable room, dry and dust free. overhead lighting and 115 volt ac power must be provided. Doors must be large enough to accommodate standard equipment racks. since most closets will contain electronic transmission equipment, heating and cooling requirements must be considered. 
DRAFT

Some rules of thumb for secondary wiring closet design and placement:

* A closet should not be expected to serve more than 50,000 square feet of office space. If space for cable access to the closet is limited, the maximum area served may be less. As an example of cable space requirements, a 3-inch diameter conduit will accommodate 50 four-pair cables.

* For telephone punch-down blocks, allow 3 linear feet of wall space for every 50,000 square feet of floor space.

* For data patch panels, allow one rack for every 100 drops. A rack should have at least 72 inches of vertical mounting space for standard, 19-inch wide panels.

* Space racks 14 inches apart to allow for cable dressing.

8.6 Closet to Work Station Cabling. Standard data cables from the secondary wiring closets to the work station outlets will consist of 24 AWG unshielded twisted pair, four pairs per cable, two cables per station. Other types of cable may be installed but they will be considered "specials". Video signals, for example, will require 75 ohm CATV coax cable. Coaxial cable will not be installed for 802.3 LANS except in cases where it has been PAGE 14 OF 25 PAGES 


\section{DRAFT}

established that length of cable runs will exceed the 100 meter limitation for unshielded twisted pair. Fiber optic cable may be required at a work station for some applications; in these cases $62.5 / 125$ fiber cables should be pulled to each station.

Each cable will terminate in a work station outlet at one end and a patch panel, cross-connect, or multiplexer in the wiring closet. This portion of the building wiring is also known as horizontal wiring because generally the secondary wiring closet is on the same floor as the work space it serves. Horizontal wiring may be run in both the ceiling and the floor in a variety of ways, each with its own advantages and disadvantages.

Ceiling systems offer a high degree of flexibility in that they offer relatively easy access to many different areas of the room below. Ceiling cable runs may be in metal raceways, inside environmental air space (plenums), or directly from closet slot to destination in any space that is available. The latter method is not as simple as it sounds, because air ducts, power wiring, lighting fixtures, etc., present numerous obstacles to be overcome. Cables are brought down from the ceiling space in wall cavities or hollow utility poles in more central parts of the room. The structural strength of the ceiling versus the combined weight of cables to be installed must be considered in planning a data-intensive space. 


\section{DRAFT}

Floor distribution systems include underfloor duct, underfloor conduit, cellular floors, and raised floors. Underfloor ducts consist of metal distribution channels, often enclosed in concrete, and metal feeder channels. Feeders intersect distribution ducts at right angles with junction boxes at the point where they meet. The junction boxes, used in pulling cable, have metal access plates that are often seen in tiled or carpeted floors. Underfloor conduit is metal pipe radiating from the wiring closet to the potential terminal location. Lower in cost, the conduit system is not as flexible as underfloor duct and should only be used in buildings with very stable terminal locations. Cellular floor systems are constructed from distribution cells and feeder ducts or trench headers; cells may be steel or concrete depending on the building structure. The cellular floor is an integral part of the structural floor, so obviously it can only be considered as a distribution system in new construction. For existing buildings, the raised floor is the most popular distribution system because of its relative ease of installation. Consisting of a series of square modules of metallic plates resting on supporting pedestals, the raised floor offers unlimited accessible space in which to conceal cables that interconnect equipment in the room. The raised floor in itself does not solve the problem of cable distribution from closet to work station. 


\section{DRAFT}

8.7. Work Station outlet.

The work station outlet is a standard point of contact between the network facilities and all terminals, telephones, PCs, printers, etc. that are connected to the network. With a planned building wiring system in place, communications devices can be added, moved, or disconnected with no disruption of the cable plant. Any reconfiguration or redirection of signals that may be required is done in the wiring closet instead of in the workspace.

The cables used in the horizontal distribution scheme terminate at a conventional 2-inch or 4-inch wide electrical outlet box wherever a flush mounted wall unit is desired. Boxes should be at least 2.75 inches deep and conform to NEMA Standard OS 11984. Hardware can also be obtained for surface mounts or floor pedestal mountings.

The faceplate of the outlet box will provide, as a minimum, two modular phone jacks that are to be used for voice and data. On this same 2-inch wide plate there is adequate space for an F-type coax connector for video and a BNC connector for the extended length 802.3 applications mentioned in the previous section. For workstations requiring 802.5 service a 4 -inch wide faceplate is 


\section{DRAFT}

used to mount the additional connector. Stations to be equipped for an FDDI dual ring network will require an additional 4-inch wide box for the two duplex optical connectors.

The modular phone jacks will both be 8-position jacks. One of the 4-pair cables entering the box will be connected to the left modular jack according to the proposed ISDN standard, thus it can be used for conventional analog telephones with eventual migration to ISDN digital equipment. The other modular jack will use six of the eight wires in the second 4-pair cable for low speed data equipment; one pair for received data, one pair for transmitted data, and one pair for "ready" signals. Pairs not assigned in this scheme should be attached to pins in the jack anyway to serve as spares for emergency use or future services.

8.8 DOCUMENTATION AND LABELING. An integral part of a successful distribution network is proper documentation. A record of all network components and cabling is absolutely essential when it becomes necessary to expand or modify the system and for effective troubleshooting. At any given time the Wiring System Administrator (see Par. 8.9) has to be able to tell which circuits are used or not used and where to find the terminal points of both. The need for this information may come up suddenly, as when a cable is inadvertently cut, hence the 
DRAFT

records must be always up to date and readily available to repair and public works personnel as well as to network managers and planners.

The documentation process must begin with' the establishment of a systematic numbering system that will uniquely identify every location where a network termination may be installed. Start the system by utilizing the building, floor and room numbers that have already been assigned. Large open areas or areas with movable partitions will require subdivision into zones; a matrix system using letters and numbers is recommended for zone labels. It is often helpful to use architectural drawings in the planning phase but be aware that the architectural numbering system is often very different from the system used by the occupants. Since the former may disappear after construction is finished, the labeling scheme should be based on the occupant's numbering system.

When numbers have been assigned they must be entered on all drawings that will be used during the installation phase. Labels for all components will be printed before installation begins so that they can be applied as each item is installed. Labels will have a standard format suited to the site, such as FFRR-ZZ-NN-XX where FFRR is floor and room, $\mathrm{ZZ}$ is zone, $\mathrm{NN}$ is component within the zone. The suffix $x x$ is optional and is used when it is 


\section{DRAFT}

necessary to designate a subcomponent such as a patch panel or chassis that is to be installed in a rack. Cables need to be labeled with location descriptions for both ends. A complete cable designation would be of the form FFRR-ZZ-NN/ffrr-zz-nn, where the first part is the source location, such as a wiring closet, and the last part is an office faceplate or similar destination. For inter-building cables, an appropriate building designator must precede the source and destination designator.

Master copies of network records are kept in a central location under the care of the Wiring System Administrator. A minimum requirement of network records is to give enough information to determine the routing of a signal path from source to destination. Copies of this minimal information, in the form of tables or charts, should be kept in the wiring closets for easy reference. Master records should contain more detailed information: type and length of cables, connector types, part numbers for terminal equipment, etc. Administrative procedures must be in place and strictly enforced for keeping these records up to date. For very small systems (500 lines or less) a simple log book may suffice; records for larger systems should be kept on an automated facility that can easily reproduce updated records on demand. 


\section{DRAFT}

8.9 Administration and Management. To ensure efficient administration and management of the base wiring system, each base should designate a Wiring system Administrator. The Wiring System Administrator will be responsible for:

* The base wiring system log, which will document the location of all circuits and switching equipment in the facility (see Section 8.8 ).

* Development of a base wiring plan which will give a master plan for upgrading the base wiring system to meet this standard.

* Assigning and allocating wiring system cable and switching resources as required to service base tenants.

* Managing and coordinating needed maintenance and the installation of new capacity.

9. IMPLEMENTATION. The implementation of an effective distribution network requires a high degree of planning, starting in the earliest stages of site and building design. The planning 


\section{DRAFT}

must begin with definition of the communications needs of the entire site and then proceed in more detail to portions of the site where facilities can be classed by building type or usage. It is important that all types of communications be included in the initial planning in order to have a truly integrated system, thus the network will serve not only the obvious voice and data processing needs but also site security, environmental control, entertainment and instructional uses.

When the communications requirements have been defined and an overall system design is in mind, enlist the aid of qualified building design personnel to help plan equipment rooms, wiring closets, cable entrances to buildings, cable ducting systems and any other items that impact architectural design. closely related issues are physical security, fire protection, electrical power distribution, lightning protection and grounding. In addition to meeting the system design goals, all of the foregoing must be implemented according to applicable building and wiring codes.

One concern that needs to be stressed emphatically during the planning phase is the allowance for future growth. Because of advancing technology and the ever increasing communications 
DRAFT

needs, a wiring system planned to accommodate only current requirements will become obsolete in a relatively short time. Installing spare conduit, extra ducts, cables, etc., is far more cost effective in the long term than to perform building retrofits.

10. RESPONSIBIIITY.

(To be filled in by NAVDAC)

11. SPECIAL INSTRUCTIONS AND COMMENTS.

a. Beneficial comments to improve this document are submitted to NAVDAC as prescribed in Attachment II.

b. The following is a list of sources of standards relating to data communications networks.

\section{ANSI}

American National Standards Institute

1430 Broadway

New York, NY 10018

(212) $642-4964$

PAGE 23 OF 25 PAGES 


\section{DRAFT}

EIA

Electronic Industries Association

2001 Eye street, NW

Washington, DC 20006

(202) $457-4900$

IEEE

Institute of Electrical and Electronic Engineers

345 East 47 th street

New York, NY 10017

(212) $705-7960$

c. Standards relating to both electric supply and communications wiring are available from:

NFPA

National Fire Protection Association

Batterymarch Park

Quincy, MA 02269

$(617) 770-3300$

NEMA

National Electrical Manufacturers Association 2101 I street

Washington, DC 20037 
DRAFT

d. Certain aspects of communications systems installation are regulated by building codes and standards, the purpose of which is to ensure quality construction and to protect life, health, and property. The major sources of building codes and standards are:

Uniform Building Code

International Conference of Building Officials

5360 South Workman Mill Ròad

Whittier, CA 90601

The BOCA Basic Building Code

Building officials and Code Administrators

International, Inc.

17926 South Halsted Street

Homewood, IL 60430

Standard Building Code (SBC)

Southern Building Code Congress International, Inc.

900 Montclair Road

Birmingham, AL 35213 


\section{ATTACHMENT I \\ Wiring for Modular Jack Receptacles}

TELEPHONE RECEPTACLE

Pin No. Function Color

1

2

3

4

5

6

7

8
Spare

Spare

- Power

Signal

Signal

+ Power

Spare

spare
Wht/Brn

Wht/Grn

Wht/Orn

Blu/Wht

Wht/Blu

Orn/Wht

Grn/Wht

Brn/Wht
DATA RECEPTACLE

Pin No. Function Color

Spare Wht/Brn

Ready out Wht/Blu

+ Transmit Data Wht/orn

- Transmit Data Orn/Wht

- Receive Data Wht/Grn

+ Receive Data Grn/Wht

Ready In

Spare Blu/Wht Brn/Wht

NOTES :

1. Color notation: Solid color/tracer color.

2. Wire pairs are indicated by color reversal, e.g., wht/brn and brn/wht are a twisted pair in the cable.

3. The telephone receptacle functions shown above conform to the ISDN standard. It has the additional advantage that some popular brands of telephone equipment with a 6-pin plug may be attached directly. Consult your telephone equipment manuals for proper pair-to-pin assignment. 
NBS-114A (REV. 2-8C)

U.S. DEPT. OF COMM.

BIBLIOGRAPHIC DATA

1. PUBLICATION OR

REPORT NO.

NISTIR $88-3867$

2. Performing Organ. Report Nod 3. Publication Date

SHEET (See instructions)

4. TITLE AND SUBTITLE

REPORT ON CABLING NAVY BASES FOR VOICE, DATA AND VIDEO

APPENDIX A: Premises Wiring Plan for Data, Voice and Video

5. $A \cup T H O R(S)$

W. E. Burr and A. L. Koenig

6. PERFORMING ORGANIZATION (If joint or other than NBS, see instructions)

7. Coneract Grant No.

NATIONAL BUREAU OF STANDARDS

U.S. DEPARTMENT OF COMMERCE

8. Type of Report \& Period Covered

GAITHERSBURG, MD 20899

9. SPONSORING ORGANIZATION NAME AND COMPLETE ADDRESS (Street, City, Stote, ZIP)

10. SUPPLEMENTARY NOTES

Document describes a computer program; SF-185. FIPS Software Summary, is attached.

11. ABSTRACT (A 200-word or less factual summary of most significant information. If document includes o significant bibliography or literature survey. mention it here)

This document proposes a common approach to wiring Navy bases for voice, data and video. Having a structured cabling plan will simplify installation of new equipment, expedite relocation of existing equipment and reduce connectivity problems between different kinds of equipment.

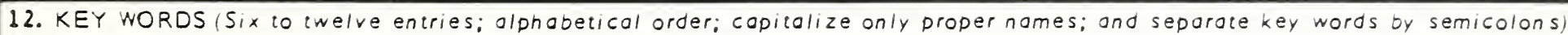
communication networks, wiring for data voice and video \& ISDN.

13. AVAILABILITY

Unlimited

For Official Distribution. Do Not Release to NTIS

Order From Suderintendent of Documents, U.S. Government Printing Office, Washington. D.C. 20402 .

15. Price

14. NO. OF PRINTED PAGES

Order From National Technical Information Service (NTIS), Springfield, VA. 2216I 

. 
